\title{
'À La Carte' Cyclic Hexapeptides: Fine Tuning Conformational Diversity while Preserving the Peptide Scaffold.
}

\author{
Sonia Ciudad, ${ }^{[a]}$ Núria Bayó-Puxán, ${ }^{[a]}$ Monica Varese, ${ }^{[a]}$ Jesús Seco, ${ }^{[a]}$ Meritxell Teixidó, ${ }^{[a]}$ Jesús \\ García, ${ }^{[a]}$ and Ernest Giralt ${ }^{*[a],[b]}$
}

\begin{abstract}
Cyclic peptides have recently emerged as promising modulators of challenging protein-protein interactions. Here we report on the design, synthesis and conformational behavior of a small library composed of C2 symmetric cyclic hexapeptides of type c(Xaa-DPro-Yaa)2, where Xaa and Yaa are chosen from alanine, isoleucine, serine, glutamic acid, arginine and tryptophan due to the favorable properties of the side chains of these residues to recognize complex protein surfaces. We used a combination of nuclear magnetic resonance and molecular dynamic simulations to perform an extensive conformational analysis of a representative set of cyclic hexapeptides. Our results indicated that both the chemical nature and the chirality of the variable Xaa and Yaa positions play an important role in the cis/trans configuration of the Xaa-D-Pro bonds and in the conformational preferences of this family of peptides. This structural tuning can be exploited in design strategies seeking to optimize the binding efficiency and selectivity of cyclic hexapeptides towards protein surfaces.
\end{abstract}

\section{Introduction}

Macrocycles have found broad application in chemical biology, biotechnology and pharmaceutical research. ${ }^{[1]}$ Over the last decade, particularly intense efforts have been channelled into the development of macrocycles and their use in the field of molecular recognition. ${ }^{[2]}$ Nowadays, most synthetic compounds reported to interact with proteins are small molecules that bind to well-defined cavities on the protein structure. ${ }^{[3]}$ More challenging, however, is the design of molecules with the ability to bind to the solvent-exposed protein surface and specifically recognize the large interfacial areas involved in protein-protein interactions, with the purpose of interfering in the functional activity of the target protein. ${ }^{[4]}$ The

[a] Dr. S. Ciudad, Dr. N.Bayó-Puxán, Dr. M.Varese, Dr. J. Seco, Dr. M. Teixidó, Dr. J. García and Prof. E. Giralt.

Institute for Research in Biomedicine (IRB Barcelona)

Barcelona Institute of Science and Technology

Baldiri Reixac, 10, 08028, Barcelona, Spain

E-mail: ernest.giralt@irbbarcelona.org

[b] Prof. E. Giralt.

Department of Inorganic and Organic Chemistry, University of Barcelona, Spain growing appreciation that these complex protein-protein interfaces appear to be 'undruggable' by small ligands has coincided with renewed interest in macrocycles, and cyclic peptides in particular, as potential protein binders, based on the premise that these cyclic molecules may be more suited to mimic natural biorecognition processes. ${ }^{[5]}$

Cyclic peptides have a number of advantages over their linear counterparts. ${ }^{[6]}$ For example, they show improved resistance to proteolytic degradation by exopeptidases due to the lack of both amino and carboxyl termini. Moreover, protein recognition is a conformation-dependent process, and converting linear sequences into their cyclized form is a common approach to modulate the performance of peptide sequences. A classic and elegant example is the chemical development of Cilengitide, a cyclic pentapeptide (c(Arg-GlyAsp-D-Phe-Val)) that is selective for $\alpha_{v}$ integrins and that was under investigation for the treatment of glioblastoma. ${ }^{[7]}$ Research on a variety of linear and cyclic penta- and hexapeptides containing the tripeptide sequence RGD (arginine, glycine and aspartic acid) showed that cycles inducing distinct conformations of RGD sequences were able to recognize various integrin receptors. ${ }^{[8]}$ These pioneering studies showed that the preorganization of the crucial side chains by cyclization significantly affects the functional activity of the amino acid sequences. Both the binding affinity and the selectivity of a recognition motif introduced into a cyclic peptide may increase when its bioactive conformation is included in the restrained conformational space of the cyclic molecule. In this case, cyclization reduces the entropic penalty of binding, thereby improving the binding interaction. However, cyclization often results in a significant decrease or even a total loss of activity. ${ }^{[9]}$ This occurs when the bioactive conformation of the recognition motif is excluded from the conformational space of the constrained peptide. It is also possible that, although accessible in the restricted conformational ensemble of the cycle, the bioactive conformation of the peptide is scarcely populated. Cyclic peptides still have considerable backbone mobility, but usually display rugged conformational landscapes that include a number of energy local minima that may not be 
uniformly sampled. Reaching some conformational states may involve the rare crossing of significant energy barriers. Therefore, the cyclic molecule would thermally fluctuate among a subset of states at a given temperature. These crucial considerations should not be overlooked in screening projects based on "ligand-oriented design" strategies. The chances of finding suitable protein ligands will depend on the availability of a robust synthetic approach to generate a variety of diverse molecules, combined with detailed knowledge of the conformational behavior of the molecules generated.

Motivated by these observations, we sought to synthesize a small library of $C 2$ symmetric cyclic hexapeptides of the type $c($ Xaa-D-Pro-Yaa)2 (Scheme 1 and Table S1). We hypothesized that this family of constrained cyclic peptides may have potential applications in the recognition of different types of protein surfaces. In fact, this library has recently demonstrated to be useful as a source of ligands able to recognize two completely unrelated proteins, such as the human vascular endothelial growth factor (VEGF) ${ }^{[10]}$ and the bacterial OmpA. One OmpA inhibitor selected from this library was shown to prevent bacterial infection in an in vivo model. ${ }^{[11]}$
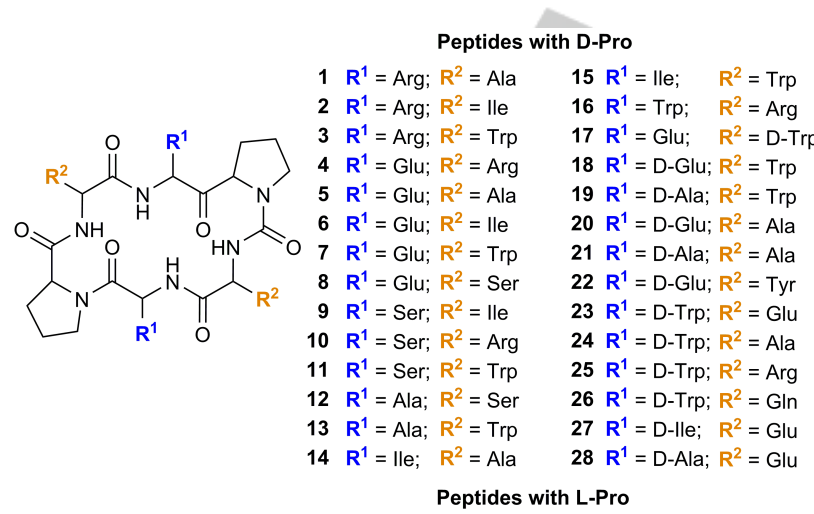

$29 \mathbf{R}^{1}=\mathrm{D}-\mathrm{Glu} ; \mathrm{R}^{2}=\mathrm{D}-\mathrm{Trp} 31 \mathbf{R}^{1}=\mathrm{Glu} ; \mathrm{R}^{2}=\mathrm{D}-\mathrm{Trp}$ $30 \mathbf{R}^{1}=\mathrm{D}-\mathrm{Glu} ; \mathrm{R}^{2}=\operatorname{Trp}$

Scheme 1. Structures of peptides 1-31. Peptides 29, 30 and 31 are the enantiomers of peptides 7,17 and 18 , respectively.

Cyclic hexapeptides are known to stabilize distinct conformations by adopting turn-like structures, although predicting their structures de novo is a considerable challenge. ${ }^{[12]}$ For this reason, we conducted an extensive conformational study combining nuclear magnetic resonance (NMR) spectroscopy and molecular dynamics (MD) simulations of a selected set of representative cyclic hexapeptides. The effect of the nature and/or chirality of the variable Xaa and Yaa positions on the configuration of the XaaD-Pro bond and on the overall conformational behavior of this family of peptides is also described in detail in this study.

\section{Results and Discussion}

\section{Design of the library of cyclic hexapeptides}

Here we aimed to synthesize a family of cyclic peptides with a preorganized structural scaffold that could optimally present a set of side chains selected for their favorable properties for protein surface recognition. We focused on C2 symmetric cyclic hexapeptides due to their straightforward synthesis and because they adopt conformations featured by two fused $\beta$-turn arrangements. ${ }^{[13]}$ Initially, we synthesized a set of peptides of the type $c($ Xaa-D-Pro-Yaa)2 (Scheme 1, peptides 1-15) including a $D$-amino acid to enhance the $\beta$-turn propensity. ${ }^{[14]}$ Xaa and Yaa could be Trp, Arg, Glu, Ser, Ala and lle. These residues were rationally selected from all proteinogenic amino acids on the basis of the physicochemical, synthetic and statistical parameters considered in our previous work. ${ }^{[15]}$

Tryptophan was chosen because it is over-represented at protein interfaces and is found on many hot spots. Like lysine, arginine ( $\mathrm{pl}=10.76$ ) displays a positive charge at physiological $\mathrm{pH}$. However, significant differences in the hydration energy and hydrogen bonding potential of these cationic amino acids make arginine more favorable to establish inter-residue contacts. Alanine is a highly abundant residue in natural systems. Since it has a small lateral side chain, it can act as a spacer for various interaction sites on peptides. Glutamic acid ( $\mathrm{pl}=3.22$ ) contributes to negative charges. Serine is a polar non-charged amino acid that outperforms threonine in solidphase peptide synthesis (SPPS). Finally, isoleucine is an aliphatic $\beta$-branched amino acid with a significantly bulky side chain. It has a higher presence in active sites than valine and leucine. Proline was selected as it is the only genetically encoded $\mathrm{N}$-alkyl amino acid and it often participates in crucial hydrophobic-hydrophobic interactions. In addition, D-proline is known to act as a turn-inducing element to promote peptide cyclization. ${ }^{[16]}$

The initial library was then expanded to include new peptides (peptides 16-31) (Scheme 1 and Table S1) with the aim of studying the influence of the chemical nature and/or chirality of the Xaa and Yaa positions on the conformational behavior of this family of cyclic peptides. 


\section{Synthesis of the C2 symmetric cyclic hexapeptides}

Cyclic hexapeptides were synthesized following a standard solid-phase Fmoc/tBu peptide synthesis protocol using 2chlorotrityl chloride (СTC) resin (Scheme 2). To compensate for the lower reactivity of the secondary amine groups of the prolyl residues, HATU was chosen as coupling reagent. All linear peptides were then synthesized as $\mathrm{H}$-Xaa-Pro-Yaa-Xaa-Pro$\mathrm{Yaa}-\mathrm{OH}$, with its respective residue chirality. To prove that no epimerization was taking place in the $C$-terminus of these peptides during the synthesis, all the corresponding $C$-terminus epimers for each peptide of the initial library were synthesized (Scheme S1). Peptide cyclization was performed in solution using PyBOP/DIEA as coupling reagent.

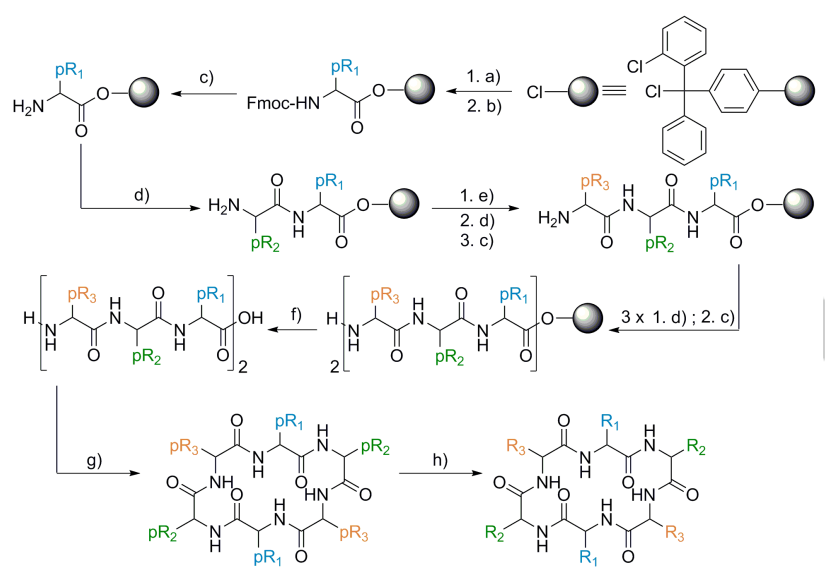

Scheme 2. Synthetic scheme for cyclic hexapeptides 1-31, where $R=$ Pro or $D-P r o$, and $R^{1}, R^{2}$ correspond to the amino acids described in Scheme 1. Reagents and conditions: a) Fmoc-AA-OH (0.7 mmol/g resin), DIPEA (10 eq.), $\mathrm{DCM}, 35 \mathrm{~min}$; b) $\mathrm{MeOH}(0.8 \mathrm{~mL} / \mathrm{g}$ resin), $15 \mathrm{~min}$; c) piperidine $20 \%$ in DMF ( $2 \times 1 \mathrm{~min}, 1 \times 10 \mathrm{~min}$ ); d) Fmoc-AA-OH (3 eq.), DIPEA (6 eq.), DMF, 35 min; e) Fmoc-AA-OH (6 eq.), DIPEA (12 eq.), DMF, 20 min; f) 1\% TFA in DCM (5 x 30 s); g) PyAOP (2 eq.), DIPEA (6 eq.), DCM/DMF (9:1) 6-12 h; and h) TFA:TIS: $\mathrm{H}_{2} \mathrm{O}(95: 2.5: 2.5), 10 \mathrm{~mL} / \mathrm{g}$ resin, $1.5 \mathrm{~h}$.

Conformational analysis of C2 symmetric cyclic hexapeptides

Effect of the chemical nature of amino acids on the conformation of $c(X a a-D-P r o-Y a a)_{2}$ cyclic hexapeptides. A representative set of cyclic hexapeptides was selected (peptides 3, 6, 7, and 11) (Scheme 1) to conduct an NMRbased conformational analysis in aqueous solution. These peptides were used to study the effects of the chemical nature (charged, polar, aromatic and $\beta$-branched) of the Propreceding and Pro-following residues on the overall conformational behavior of this family of cyclic hexapeptides.
The observation of three individual spin systems in the NMR spectra of all four peptides confirmed the C2 symmetric nature of these molecules (Figure S1). This observation is consistent with these peptides populating either a unique conformation or different states coexisting in fast equilibrium in the NMR timescale. A complete resonance assignment is provided in the Supporting Information (Table S2). The resemblance of structure-sensitive NMR parameters such as secondary chemical shifts, ${ }^{3} J_{\mathrm{NHHa}}$ values, temperature coefficients of amide protons (Table 1), and ${ }^{1} \mathrm{H}-{ }^{1} \mathrm{H}$ NOESY correlations indicated that the four peptides adopt a similar conformation in solution. The configuration of the Xaa-D-Pro bond was trans in these peptides, as indicated by the chemical shift differences between the ${ }^{13} \mathrm{C}_{\beta}$ and ${ }^{13} \mathrm{C}_{Y}\left(\Delta \delta \mathrm{C}_{\beta \gamma}\right)$ of the Pro residue, with values around $4 \mathrm{ppm}$ (Table 1). ${ }^{[17]}$ The observation of an intense $\mathrm{H}_{\alpha}(\mathrm{Xaa})-\mathrm{H}_{\delta}$ (D-Pro) NOE crosspeak in the corresponding NOESY spectra (Figure 1a) further confirmed the trans configuration of the Xaa-D-Pro bond in these four peptides.

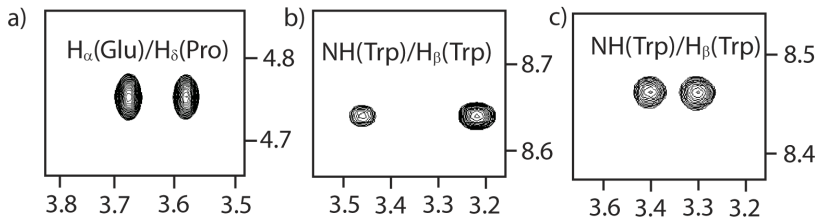

d)
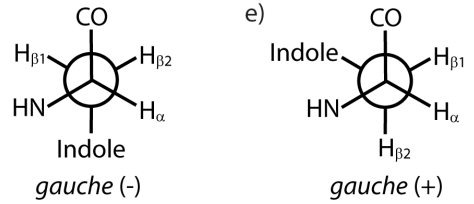

Figure 1. Expanded regions of the NOESY spectra of peptides 7 (a) and (b) and 3 (c) showing intra-residue cross-peaks between $\mathrm{H}_{\alpha}$ proton of the Glu residues and the $\mathrm{H}_{\delta}$ protons of the D-Pro residues, which confirmed the trans conformation of the Xaa-Pro bonds (a); and between the resonances of the $\mathrm{NH}$ and the $\mathrm{H}_{\beta}$ protons of the Trp residues (b) and (c). For peptide 7, the upfield $\mathrm{H}_{\beta 1}$ (Trp) proton presents a more intense NOE crosspeak with the $\mathrm{NH}(\operatorname{Trp})$ amide proton than the corresponding downfield $\mathrm{H}_{32}(\operatorname{Trp})$ proton, thereby indicating the predominance of the gauche (-) rotamer. In peptide 3 , both diastereotopic $\mathrm{H}_{\beta}(\mathrm{Trp})$ protons display NOE cross-peaks with the $\mathrm{NH}$ (Trp) amide proton of similar intensity, thus suggesting an averaged population between the gauche (-) and gauche (+) rotameric states.

The $\mathrm{NH}$ amide protons of the Xaa and Yaa residues within this set of peptides exhibited a well-differentiated trend. Irrespective of the chemical nature of the amino acid, the $\mathrm{NH}$ of Xaa residues showed higher-field chemical shifts (Table S2) and 
smaller temperature coefficient values $\left(-\Delta \bar{\delta}_{\mathrm{NH}} / \Delta T\right)$ than those of the Yaa residues (Table 1).

The reduced temperature dependence of the Xaa amide protons, ranging from $1.0 \mathrm{ppb} \mathrm{K}^{-1}$ in peptide 11 (Xaa = Ser) to $4.5 \mathrm{ppb} \mathrm{K}^{-1}$ in peptide 3 (Xaa = Arg), indicates their involvement in intramolecular hydrogen bonds. In contrast, the $\mathrm{NH}$ protons of the Yaa residues showed large temperature coefficients $\left(7.7-8.5 \mathrm{ppb} \mathrm{K}^{-1}\right)$ characteristic of solvent-exposed backbone amides. The ${ }^{3} J_{\mathrm{NHHa}}$ values observed for these peptides were in the range $7.7-9.6 \mathrm{~Hz}$, consistent with a $\beta$-like conformation (Table 1). All these results, combined with the detection of a strong $\mathrm{H}_{\alpha}$ (D-Pro) $\mathrm{NH}$ (Yaa) NOE cross-peak (Figure S2), is consistent with a symmetric backbone conformation containing two fused type II' $\beta$-turns. Regarding side chain conformation, interestingly, the two methylene $\mathrm{H}_{\beta}$ protons of the aromatic side chain in Trpcontaining peptides (3, 7 and 11) showed marked differential NMR behavior. Peptides 7 (c(Glu-D-Pro-Trp)2) and 11 (c(SerD-Pro-Trp)2) presented a noticeable diastereotopic $\mathrm{H}_{\beta}$ (Trp) splitting (3.48 and 3.22 ppm for peptide 7; and 3.49 and 3.22 ppm for peptide 11) and a remarkable difference in ${ }^{3} \mathrm{~J}_{\mathrm{HH \beta}}(\mathrm{Trp})$ values ( 4.3 and $9.5 \mathrm{~Hz}$ for peptide 7 ; and 4.4 and $9.6 \mathrm{~Hz}$ for peptide 11) (Figure 2). The pronounced differences observed between diastereotopic $\mathrm{H}_{\beta}$ (Trp) protons suggest that the aromatic side chain in these peptides is relatively fixed. Of the two $\mathrm{H}_{\beta}$ (Trp) protons, the one with the largest ${ }^{3} \mathrm{~J}_{\mathrm{HaH} \beta}$ (Trp) value $\left(\delta_{\mathrm{H} \beta}=3.22 \mathrm{ppm}\right)$ was also the one showing the most intense intra-residue NOE cross-peak with the amide $\mathrm{NH}$ (Trp) proton (Figure 1b).

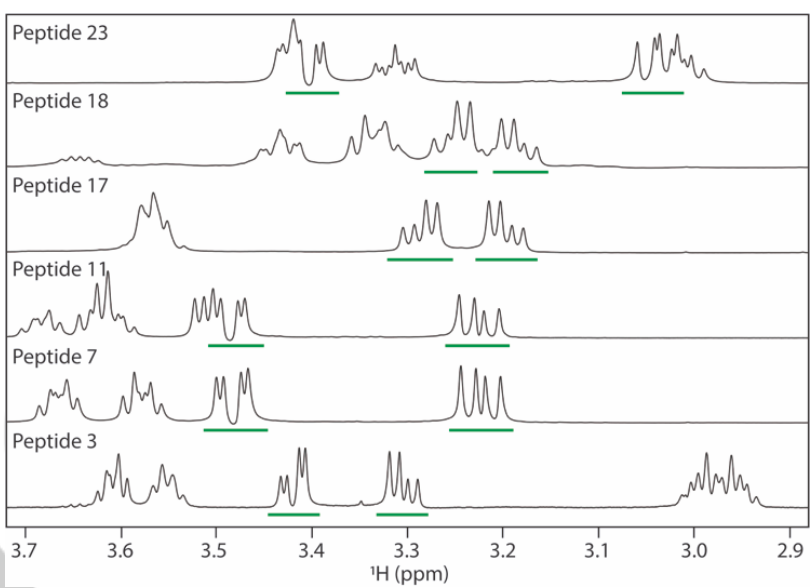

Figure 2. Partial ${ }^{1} \mathrm{H}-\mathrm{NMR}$ spectra of peptides $3,7,11,17,18$ and 23 showing the differential NMR behavior of the diastereotopic methylene $H_{\beta}(\operatorname{Trp})$ protons (underlined).

This observation would suggest the predominance of the gauche (-) rotamer (Figure 1d). Most probably, the rotation of the Trp side chain is restricted by the presence of $\mathrm{CH}-\pi$ interactions between the aromatic ring and the Pro methylene groups. The spatial proximity of the Pro and Trp side chains is in agreement with the upfield shift of $\mathrm{H}_{\alpha}$ (Pro) (4.21 ppm in both peptides) and $\mathrm{H}_{\beta}$ (Pro) protons (1.93 and 1.44 ppm for peptide 7; and 1.91 and $1.42 \mathrm{ppm}$ for peptide 11), ${ }^{[18]}$ when compared with the non-Trp containing peptide $6\left(\delta_{\mathrm{Ha}}(\right.$ Pro $)=4.47 \mathrm{ppm}$ and $\delta_{H \beta}($ Pro $)=2.25$ and $\left.1.92 \mathrm{ppm}\right)$. To support the NMR analysis, molecular dynamics (MD) simulations of peptide 7 were conducted.

Table 1. Summary of NMR data and Xaa-D-Pro bond conformation of peptides 3, 6, 7, 11, 16, 17, 18 and 23. b.s. means broad signal.

\begin{tabular}{|c|c|c|c|c|c|c|c|}
\hline \multirow{2}{*}{ Peptide } & \multirow{2}{*}{$\begin{array}{c}\text { Sequence } \\
\mathrm{c}(\text { Xaa-D-Pro-Yaa })_{2}\end{array}$} & \multirow{2}{*}{$\begin{array}{c}\Delta \delta C_{\beta \gamma} \text { Xaa-D-Pro } \\
\text { bond (ppm) }\end{array}$} & \multirow{2}{*}{$\begin{array}{l}\text { Xaa-D-Pro bond } \\
\text { conformation }\end{array}$} & \multicolumn{2}{|c|}{$\begin{array}{c}-\Delta \delta_{\mathrm{NH}} / \Delta T \\
(\mathrm{ppb} / \mathrm{K})\end{array}$} & \multicolumn{2}{|c|}{${ }^{3} J_{\mathrm{NHH \alpha}}(\mathrm{Hz})$} \\
\hline & & & & Xaa & Yaa & Xaa & Yaa \\
\hline 3 & c(Arg-D-Pro-Trp $)_{2}$ & 3.8 & trans/trans & 4.5 & 8.5 & 8.5 & b.s. \\
\hline 6 & $c(\text { Glu-D-Pro-Ile })_{2}$ & 4.2 & trans/trans & 3.5 & 9.0 & 9.0 & 8.2 \\
\hline 7 & c(Glu-D-Pro-Trp $)_{2}$ & 3.7 & trans/trans & 1.5 & 8.1 & 8.1 & 7.7 \\
\hline 11 & $c(\text { Ser-D-Pro-Trp })_{2}$ & 3.6 & trans/trans & 1.0 & 8.7 & 8.7 & 7.7 \\
\hline 16 & $c(\text { Trp-D-Pro-Arg })_{2}$ & 4.1 & trans/trans & 2.8 & 9.6 & 9.6 & b.s. \\
\hline 17 & $c(\text { Glu-D-Pro-D-Trp })_{2}$ & 5.8 & trans/trans & 8.7 & 2.7 & 5.3 & 7.7 \\
\hline 18 & $c(\text { D-Glu-D-Pro-Trp })_{2}$ & 10.2 & cis/cis & 5.0 & 7.4 & 7.4 & 6.5 \\
\hline & & 3.1 & trans/trans & 8.0 & 8.6 & 8.6 & 6.4 \\
\hline 23 & $c(\mathrm{D}-\text { Trp-D-Pro-Glu })_{2}$ & 9.6 & cis/cis & 7.0 & 6.6 & 6.6 & 6.8 \\
\hline
\end{tabular}


In perfect agreement with the experimental results, the conformers obtained by replica exchange molecular dynamics (REMD) suggested a well-defined C2 symmetric conformation with two fused type II' $\beta$ turns, with D-Pro occupying the $i+1$ position, and hydrogen bonds between the $\mathrm{i}$ and $\mathrm{i}+3$ positions (Figure S3). The REMD-derived Ramachandran plots for these peptides (Figure S4) correlate with those of a $\beta$ II' turn $(\Phi(i+1)$ $\left.=60^{\circ}, \Psi(\mathrm{i}+1)=-120^{\circ} ; \Phi(\mathrm{i}+2)=-80^{\circ}, \Psi(\mathrm{i}+2)=0^{\circ}\right){ }^{[19]}$ Also consistent with the NMR results, the REMD simulations of peptide 7 indicated that the side chain $X^{1}$ dihedral angle samples a major population (Figure S5a) corresponding to the gauche $(-)$ rotamer $\left(X^{1} \sim-60^{\circ}\right) .{ }^{[20]}$ The spatial proximity between the Trp and Pro side chains also pointed to the occurrence of $\mathrm{CH}-\mathrm{m}$ interactions (Figure $\mathrm{S} 5 \mathrm{~b}$ ).

In the case of peptide 3 (c(Arg-D-Pro-Trp) 2 ), NMR and REMD analysis indicated that the Trp side chains appeared to have different rotameric preferences. This peptide displayed a significant difference in ${ }^{3} \mathrm{~J}_{\mathrm{HH} \beta}$ values for the $\mathrm{H}_{\beta}(\operatorname{Trp})$ protons $(5.3$ and $8.3 \mathrm{~Hz}$ ) but a smaller diastereotopic splitting of these methylene protons (3.42 and $3.30 \mathrm{ppm}$ ) than peptides 7 and 11 (Figure 2). Moreover, in peptide 3 both $\mathrm{H}_{\beta}$ (Trp) protons exhibited $\mathrm{NH}-\mathrm{H}_{\beta}$ intra-residue $\mathrm{NOE}$ cross-peaks of similar intensity (Figure 1c).

Analysis of the REMD simulations confirmed that, like peptide 7, peptide 3 adopts a $C 2$ symmetric conformation with two fused type II' $\beta$-turns (Figure S3). However, the Trp side chain of peptide 3 sampled two similarly populated rotameric states with $X^{1}$ values around 60 and $-60^{\circ}$ (Figure S5c) corresponding to the gauche $(+)$ and gauche (-) rotamers, respectively (Figure $1 \mathrm{~d}$ and $1 \mathrm{e}$ ). ${ }^{[20]}$ The smaller diastereotopic splitting of the $\mathrm{H}_{\beta}$ (Trp) protons and closer ${ }^{3} \mathrm{~J}_{\mathrm{HaH} \beta}$ values for peptide 3 when compared with peptide 7 are likely to be the effect of the conformational average of the two different Trp rotamers observed for 3 . Furthermore, the most populated rotamer, gauche $(+)$, would place the Trp and Arg side chains fairly close, thereby suggesting the presence of cation- $\pi$ interactions that may fix the Trp side chain (Figure S5d).

To assess the influence of the position of the aromatic residue on Trp side chain interactions, we examined peptide 16 (c(Trp-D-Pro-Arg) $)_{2}$ ), in which the Trp residue precedes the D-Pro. The ${ }^{3} J_{\mathrm{NHHa}}$ values, temperature coefficients of the amide protons and the $\Delta \delta C_{\beta \gamma}$ (D-Pro) values (Table 1) suggested that peptide 16 also adopts a conformation with two type II' $\beta$-turns, similar to that previously described for peptides 3, 6, 7 and 11 .
However, peptide 16 did not show evidence of restricted rotation of the Trp side chain. The two $\mathrm{H}_{\beta}$ (Trp) protons displayed similar chemical shifts $\left(\delta_{\mathrm{H} \beta}=3.31\right.$ and $\left.3.26 \mathrm{ppm}\right)$ and ${ }^{3} \mathrm{~J}_{\mathrm{HaH \beta} \beta}$ values $(\sim 7.5 \mathrm{~Hz})$, which suggest rapid flipping of the aromatic side chain, thereby indicating the presence of distinct rotamers in fast exchange in the NMR time-scale.

In addition, the chemical shifts of the D-Pro protons did not appear to be affected by the aromatic ring $\left(\delta_{\mathrm{H} \alpha}=4.27 \mathrm{ppm} ; \delta_{\mathrm{H} \beta}\right.$ $=2.03$ and $1.80 \mathrm{ppm}$ ). This observation supports the notion of the absence of strong Trp/Pro intramolecular interactions. In conclusion, cyclic hexapeptides of the type c(Xaa-D-Pro-Yaa $)_{2}$ adopt a $C 2$ symmetric all-trans conformation with two fused type II' $\beta$-turns. When D-Pro is followed by a Trp residue, the aromatic side chain seems to participate in structure-stabilizing intramolecular interactions, either with the preceding Pro $(\mathrm{CH}-$ $\pi$ interactions) or with the following Arg (cation- $\pi$ ), resulting in the predominance of specific Trp rotamers.

Influence of the chirality of amino acids on the conformation of $c$ (Xaa-D-Pro-Yaa) ${ }_{2}$ cyclic hexapeptides. To examine how the stereochemistry of each position on the repeated tripeptide motif present in this family of cyclic hexapeptides affects the conformational behavior of the cycle, all possible heterochiral stereoisomers of peptide 7 (c)(Glu-DPro-Trp)2) were synthesized (peptides 17, 18 and 29-31) (Scheme 1). Nonetheless, it was not possible to obtain the allL (c(Glu-Pro-Trp) 2 and all-D c(D-Glu-D-Pro-D-Trp) $)_{2}$ ) homochiral stereoisomers as hexapeptides. Instead, during the cyclization, the formation of the corresponding cyclic homochiral dodecapeptides (c(Glu-Pro-Trp)4 and c(D-Glu-D-Pro-D-Trp) 4 ) was only observed. Peptides 29 (DLD chirality), 30 (DLL chirality) and $\mathbf{3 1}$ (LLD chirality) are the enantiomeric sequences of peptides 7 (LDL chirality), 17 (LDD chirality) and 18 (DDL chirality), respectively, as confirmed both by CD (Figure S6), and NMR (Figure S7).

Therefore, an NMR-based conformational analysis of peptides 17 (LDD chirality) and 18 (DDL chirality) was conducted and the results were compared with those of peptide 7 (LDL chirality). A complete resonance assignment for these peptides is provided in the Supporting Information (Table S3). As expected, peptide 17 (c(Glu-D-Pro-D-Trp)2) showed C2 symmetry in the NMR time-scale. The $\Delta \delta \mathrm{C}_{\beta \gamma}$ (D-Pro) value obtained for this peptide $\left(\Delta \delta \mathrm{C}_{\beta \gamma}=5.8 \mathrm{ppm}\right)$ indicates prolyl imide bonds in trans configuration. Peptides 17 (LDD chirality) and 7 (LDL chirality) showed an opposite $\mathrm{NH}$ orientation. In 
peptide 7, the amide $\mathrm{NH}$ proton of Pro-preceding L-Glu residues showed upfield chemical shifts $\left(\delta_{\mathrm{NH}}=7.70 \mathrm{ppm}\right)$ and seemed to be solvent-protected $\left(-\Delta \delta_{\mathrm{NH}} / \Delta T=1.5 \mathrm{ppb} \mathrm{K}{ }^{-1}\right)$, whereas the amide $\mathrm{NH}$ proton of Pro-following L-Trp residues resonates at $8.48 \mathrm{ppm}$ and are solvent-exposed $\left(-\Delta \delta_{\mathrm{NH}} / \Delta T=\right.$ $8.1 \mathrm{pp} \mathrm{K}^{-1}$ ). In peptide 17 , in contrast, while the amide $\mathrm{NH}$ proton of Pro-following D-Trp residues displayed upfield chemical shifts (7.24 ppm) and appeared to be H-bonded ($\left.\Delta \delta_{\mathrm{NH}} / \Delta T=2.7 \mathrm{ppb} \mathrm{K}^{-1}\right)$, the Pro-preceding L-Glu residues $\left(\delta_{\mathrm{NH}}\right.$ $=8.20 \mathrm{ppm})$ displayed values $\left(-\Delta \delta_{\mathrm{NH}} / \Delta T=8.7 \mathrm{ppb} \mathrm{K}^{-1}\right)$ typical of solvent-exposed amides (Table 1). This change in $\mathrm{H}$ bonding with respect to peptide 7 also implies a shift of the DPro position in the $\beta$-turn. Assuming that D-Trp residues of peptide 17 are $\mathrm{H}$-bonded and occupy the i position of the $\beta$ turn, the D-Pro would be found in the $\mathrm{i}+2$ position. D-Pro restricts the backbone dihedral angle $\varphi$ to $60 \pm 20^{\circ}$. ${ }^{21]}$ This range of values for the $\varphi(i+2)$ dihedral is possible only in the type II $\beta$-turn suggesting that peptide 17 has a high population of this $\beta$-turn type in solution.

Comparing the NMR spectra of peptide 17 (LDD chirality) and 7 (LDL chirality), a general observation emerges, namely that the former peptide displays higher conformational flexibility. As an example, the chemical shift difference $\Delta \delta \mathrm{C}_{\beta \gamma}$ (D-Pro) of peptide $17\left(\Delta \delta \mathrm{C}_{\beta \gamma}=5.8 \mathrm{ppm}\right)$ was slightly larger than that of peptide $7\left(\Delta \delta \mathrm{C}_{\beta \gamma}(\mathrm{D}-\mathrm{Pro})=3.6 \mathrm{ppm}\right)$, thereby suggesting an increased flexibility of proline rings in peptide 17. A further suggestion of the dynamic nature of peptide 17 is the observation that the chemical shift of the $\mathrm{H}_{\delta}$ (Pro) proton resonating at $3.58 \mathrm{ppm}$ was found to be temperaturedependent (Figure S8). In the temperature range tested (278 to $308 \mathrm{~K}$ ), this behavior was detected only in peptide 17 . The ${ }^{3} \mathrm{JNHa}_{\mathrm{NHa}}$ values for peptide $17(5.3$ and $7.7 \mathrm{~Hz})$ were smaller than those observed for peptide $7\left(8.1\right.$ and $7.8 \mathrm{~Hz}$ ) (Table 1). ${ }^{3} \mathrm{JNH}_{\mathrm{NH}}$ values around $6-7 \mathrm{~Hz}$ are indicative of the coexistence of rapidly interconverting conformers in solution.

Another notable difference of peptide 17 (c)(Glu-D-Pro-D$\operatorname{Trp})_{2}$ ) with respect to peptides of the series $\mathrm{c}($ Xaa-D-Pro-Trp)2 is that the NMR spectra of peptide 17 did not show evidence of the predominance of any Trp rotamer. Similar $\mathrm{H}_{\beta}$ (Trp) chemical shifts (3.29 and $3.21 \mathrm{ppm}$ ) and ${ }^{3} \mathrm{~J}_{\mathrm{HaH \beta}}$ (Trp) values $(7.2$ and $7.0 \mathrm{~Hz}$ ) were observed for the diastereotopic methylene $\mathrm{H}_{\beta}$ (Trp) protons of this peptide (Figure 2).

Unlike peptide 7 , analysis of the REMD trajectories of peptide 17 suggested the occurrence of two predominant backbone conformations (Figure S9). Dihedral angles for Pro residues are around $\Phi=60 \pm 20^{\circ}$ and $\Psi=0 \pm 30^{\circ}$, indicating either type $\beta \mathrm{I}^{\prime}$ (canonical dihedral values $\Phi(\mathrm{i}+1)=60^{\circ}, \Psi(\mathrm{i}+1)$ $\left.=30^{\circ}\right)^{[19]}$ or type $\beta$ II (canonical dihedral angles $\Phi(i+2)=80^{\circ}, \Psi$ $\left.(i+2)=0^{\circ}\right) \cdot{ }^{[19]}$ If type I' $\beta$-turns with $D$-Pro in the $i+1$ position were present in the structural ensemble of peptide 17 , the $i+2$ position would be occupied by $\mathrm{D}$-Trp residues displaying typical $\beta l^{\prime}$ dihedral angles $\left(\Phi(i+2)=90^{\circ}, \Psi(i+2)=0^{\circ}\right)$. These values perfectly correlate with the most populated region observed in the Ramachandran plot of D-Trp (Figure S10). In the case of type II $\beta$-turns, Glu residues would occupy $i+1$ positions, presenting typical II $\beta$-turn dihedral angles $(\Phi(i+1)=$ $\left.-60^{\circ}, \Psi(i+1)=120^{\circ}\right)$, which are in agreement with one of the regions sampled by Glu residues in the Ramachandran plot. These observations may indicate that the conformational flexibility of peptide 17 suggested by NMR is most likely the result of a fast interconversion process, on the NMR time scale, between type II and type I' $\beta$-turn populations. On the basis of metadynamics simulations, a possible mechanism for this type I' $\leftrightarrow$ II inter-conversions in cyclic hexapeptides was recently reported by McHugh et al. ${ }^{[22]}$ The authors proposed a pathway that involves a shift of the $\beta$-turn locations. The $i+2$ residues of the initial $\beta$-turn assume the $i+1$ positions of $\beta$-turns in the final conformation via coupled two-dihedral changes.

NMR analysis of peptide 18 (c(D-Glu-D-Pro-Trp)2) was complicated since it presented various conformers in slow exchange in the NMR time-scale (Figure 3). Remarkably, the major species $(85 \%$ at $298 \mathrm{~K})$ corresponded to the C2 symmetric two-cis D-Glu-D-Pro conformer, as deduced from the $\Delta \delta \mathrm{C}_{\beta \gamma}$ (D-Pro) value $\left(\Delta \delta \mathrm{C}_{\beta \gamma}=10.2 \mathrm{ppm}\right)$. The $C 2$ symmetric all-trans conformer was also identified on the basis of the $\Delta \delta \mathrm{C}_{\beta y}$ (D-Pro) value $\left(\Delta \delta \mathrm{C}_{\beta \gamma}=3.1 \mathrm{ppm}\right)$. A subset of minor NMR signals coexisting with those of the two major species was also observed but they could not be assigned due to their lower intensity and to severe spectral overlap. A recent study ${ }^{[23]}$ reported that a peptide with the enantiomeric chirality of peptide 18, c(Ser-Pro-D-Phe-Lys-Pro-D-Phe), adopts a conformation with two pseudo-type Vlb $\beta$-turns. This type Vlb $\beta$-turn is characterized by cis proline in position $i+2$ and no intramolecular hydrogen bonds. These features are in agreement with the NMR experimental data obtained for peptide 18 (Table 1). Analysis of the REMD simulations of the major conformer of peptide 18 (two-cis D-Glu-D-Pro bonds) supported the presence of $\beta \mathrm{Vlb}$-like turns. 
a)

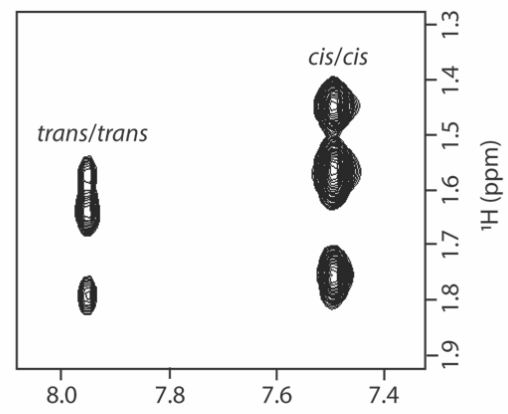

b)

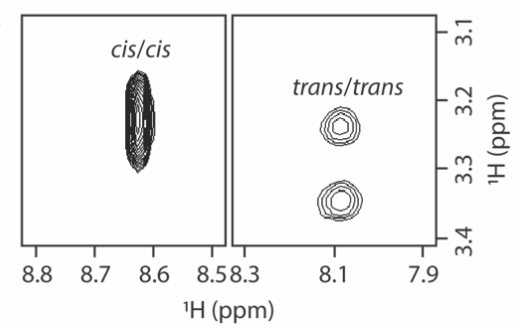

Figure 3. Expanded regions of the TOCSY spectra of peptide 18 showing the presence of different spin systems for Glu (a) and Trp (b) residues corresponding to the trans/trans $(\sim 15 \%)$ and cis/cis ( 85\%) conformations observed for this peptide.

Thus, as expected for this type of turn, no hydrogen bonds were observed in the most populated states of the trajectory (Figure S11). Most importantly, the REMD-derived Ramachandran plots (Figure S12) displayed values characteristic of the enantiomer of a $\beta$ VIb-like turn with D-Glu residues in the $\mathrm{i}+1$ position $\left(\Phi=135^{\circ}, \Psi=-135^{\circ}\right)$ and D-Pro in the $\mathrm{i}+2$ position $\left(\Phi=75^{\circ}, \Psi=-160^{\circ}\right)$. A surprising observation, however, is that distinct Ramachandran plots were obtained for the same type of residue, for example between the two D-Pro residues. One of the D-Pro residues sampled a region corresponding to $\Phi=60^{\circ}, \Psi=0^{\circ}$, which may point to the presence of pseudo- $\beta$ Vla2 turns. These observations may indicate the presence of non-symmetric conformations, which may correspond to the minor species detected in the ${ }^{1} \mathrm{H}$ NMR spectrum of this peptide.

Study of the cis content of peptides of the type (D-Xaa-D-Pro$\underline{\mathrm{Yaa}})_{2}$

The cis Xaa-Pro segment of type VI $\beta$-turns has been proposed as a potential binding motif for various peptidyl prolyl cis/trans isomerases (PPlases), which are emerging as important biomedical targets related with diseases such as cancer, Alzheimer's disease, and asthma. ${ }^{[24]}$ Given that peptide 18 (DDL chirality) seemed to adopt, at least to some extent, a double $\beta$ Vlb-like structure, we performed several modifications on this peptide to assess the influence of the Pro-preceding and Pro-following positions on the configuration of the D-XaaD-Pro bond. First, the Xaa and Yaa positions of peptide 18 were individually (peptides 19 and 20) and simultaneously (peptide 21) (Scheme 1) replaced by Ala while retaining the configuration of the parent compound. The Trp residues were then replaced for Tyr (peptide 22) (Scheme 1) to compare the effects of different aromatic residues in the Yaa position. A complete resonance assignment for these peptides is provided in the Supporting Information (Table S4 and Figure S13).

The NMR-derived populations of conformers at $298 \mathrm{~K}$ are shown in Table 2. Removal of the aromatic side chain of the Yaa position reduced the cis/cis content from $85 \%$ in peptide 18 to $60 \%$ in peptide 20 . More interestingly, when the Xaa and Yaa residues were simultaneously replaced by Ala (peptide 21), the cis/cis content was drastically reduced to $<5 \%$. No significant changes on cis/cis content were observed between peptides $18($ Yaa $=$ Trp $)$ and $22($ Yaa $=$ Tyr $)$. These results indicate that the cis D-Xaa-D-Pro bond is favored when the DPro residue is followed by aromatic amino acids. However, the nature of the amino acid placed in the Xaa position also affects the conformation of the D-Xaa-D-Pro bond.

Next, we synthesized four cyclic hexapeptides containing a D-Trp-D-Pro motif followed by a variable Yaa position (peptides 23-26) (Scheme 1 and Table S5). All the evaluated peptides, irrespective of the chemical nature of the Yaa residue (23, Yaa = Glu; 25, Yaa = Arg; and 26, Yaa = Gln) exhibited a D-Xaa-D-Pro bond cis/cis content in excess of 95\% (Table 2 and Figure S14). Of particular interest is the comparison between peptides 23 (C(D-Trp-D-Pro-Glu)2) and 18 (c(D-Glu-DPro-Trp) 2 ), in which the nature of the residues on Xaa and Yaa positions were switched. Remarkably, the cis/cis content increased from $85 \%$ in peptide 18 to $>95 \%$ in peptide $\mathbf{2 3}$, thereby demonstrating that the tendency of aromatic residues to favor cis D-Xaa-D-Pro bonds increases when they precede D-Pro residues. This observation is in agreement with a previous report that proposed that aromatic side chains preceding D-Pro residues tend to favor the cis conformation of amide bonds. ${ }^{[25]}$ 
Table 2. Cis/cis content at $298 \mathrm{~K}$ of the D-Xaa-D-Pro bonds in peptides 1923.

\begin{tabular}{|c|c|c|c|}
\hline Peptide & Sequence & $\begin{array}{c}\text { cis/cis } \\
\text { content }^{[c]}\end{array}$ & $\begin{array}{c}\text { Other } \\
\text { conformers } \\
\text { detected }\end{array}$ \\
\hline 18 & c(D-Glu-D-Pro-Trp) & $\sim 85 \%$ & trans/trans \\
\hline $19^{[a]}$ & $c(\text { D-Ala-D-Pro-Trp })_{2}$ & -- & -- \\
\hline 20 & $c(\text { D-Glu-D-Pro-Ala })_{2}$ & $\sim 60 \%$ & trans/trans \\
\hline 21 & $\mathrm{c}(\mathrm{D}-\mathrm{Ala}-\mathrm{D}-\mathrm{Pro}-\mathrm{Ala})_{2}$ & $<5 \%$ & trans/trans \\
\hline 22 & $c(\text { D-Glu-D-Pro-Tyr })_{2}$ & $\sim 90 \%$ & trans/trans \\
\hline $23^{[b]}$ & $c(\text { D-Trp-D-Pro-Glu })_{2}$ & $>95 \%$ & -- \\
\hline $24^{[a]}$ & c(D-Trp-D-Pro-Ala $)_{2}$ & - & - \\
\hline $25^{[b]}$ & $c(D-T r p-D-P r o-A r g) 2$ & $>95 \%$ & -- \\
\hline $26^{[b]}$ & $c(D-T r p-D-P r o-G I n) 2$ & $>95 \%$ & \\
\hline 27 & $c(\text { D-Ile-D-Pro-Glu })_{2}$ & $\sim 80 \%$ & cis/trans \\
\hline 28 & $c(\text { D-Ala-D-Pro-Glu })_{2}$ & $\sim 10 \%$ & trans/trans \\
\hline
\end{tabular}

[a] Peptides 19 and $\mathbf{2 4}$ could not be evaluated due to solubility restrictions. [b] Minor conformation could not be unambiguously assigned due to very low signal intensity.

[c] Quantification was done by peak integration in $1 \mathrm{D}{ }^{1} \mathrm{H}-\mathrm{NMR}$ spectra. More precise quantification could not be performed due to signal overlap.

To further analyze the influence of the aromatic side chain in the Pro-preceding position, we compared the cis/cis content of peptide 23 (c(D-Trp-D-Pro-Glu $)_{2}$ ) with two additional peptides in which the D-Trp was replaced by D-lle (peptide 27 ) and by D-Ala (peptide 28) (Table S5).

Removal of the aromatic side chain decreased the cis/cis content in both cyclic hexapeptides (Table 2). Replacement of the aromatic residue by Ala had a drastic effect. In peptide $\mathbf{2 8}$ we observed only $10 \%$ of the two-cis conformer, the all-trans being the most abundant. However, when the aromatic residue was substituted by a bulky side chain, such as lle (peptide 27), only a moderate decrease in the cis/cis content (from $>95 \%$ to $80 \%$ ) was observed. These findings suggest that the cis configuration of the amide bond in D-Xaa-D-Pro motifs is favored to minimize steric effects when bulky side chains precede the D-Pro residue.

The major difference in the NMR behavior of peptides 18 $\mathrm{C}$ (D-Glu-D-Pro-Trp $)_{2}$ and $23 \mathrm{c}(\mathrm{D}-\mathrm{Trp}-\mathrm{D}-\mathrm{Pro}-\mathrm{Glu})_{2}$ is that the latter exhibited restricted rotation of the aromatic side chains, as deduced from the ${ }^{1} \mathrm{H}_{\beta}$ chemical shifts (3.39 and 3.03 ppm)
(Figure 2) and ${ }^{3} \mathrm{~J}_{\mathrm{HaH \beta}}$ coupling constants $(4.4$ and $10.9 \mathrm{~Hz}$ ) of Trp residues. The $\mathrm{H}_{\alpha}$ (Pro) proton of peptide $23\left(\delta_{H \alpha}=3.43\right.$ ppm) showed the largest upfield shift of all the peptides described in this work, which may provide indirect evidence of intramolecular $\mathrm{CH}-\pi$ interactions.

The observation of NOE crosspeaks of similar intensities between $\mathrm{NH}(\operatorname{Trp})-\mathrm{H}_{\beta 1} / \mathrm{H}_{\beta 2}(\operatorname{Trp})$ (Figure 4) suggested the presence of a major trans rotamer for this side chain. The same behavior was observed for peptides 25 and 26, as reflected by the observed diastereotopic splitting of $\mathrm{H}_{\beta}$ (Trp) protons $\left(\delta_{H \beta}=3.38,3.01 \mathrm{ppm}\right.$, and 3.40, $3.05 \mathrm{ppm}$ for peptides 25, and 26, respectively), the ${ }^{3} J_{\mathrm{HaH \beta}}(\operatorname{Trp})$ coupling constants ( ${ }^{3} J_{\mathrm{HaH \beta}}=4.8$ and $10.4 \mathrm{~Hz}$ in both peptides) and the upfield shift on the $\mathrm{H}_{\alpha}$ (Pro) protons ( $\delta_{\mathrm{Ha}}=3.48$ and $3.45 \mathrm{ppm}$ for peptides 25, and 26, respectively).
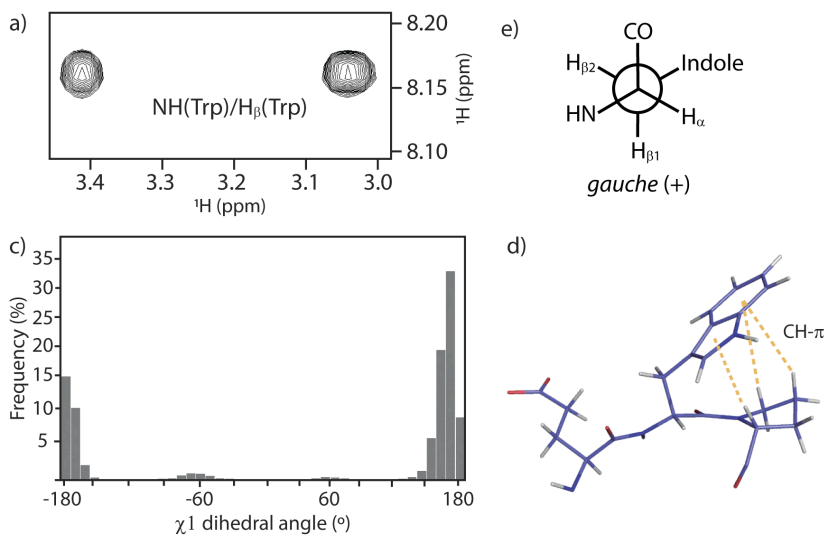

Figure 4. a) Selected region of the NOESY spectrum of peptide 23 showing intra-residue NOE correlation for the Trp residues of the trans conformer. Both $\mathrm{H}_{\beta}$ protons exhibited $\mathrm{NH}-\mathrm{H}_{\beta}$ intra-residue NOE cross-peaks of similar intensity. b) Representation of the Newman projection of the trans rotamer. c) Histogram representation of the values sampled by the $X 1$ dihedral angle in the REMD simulation for peptide 23; and d) Schematic representation showing $\mathrm{CH}-\pi$ interactions between the side chains of proline and tryptophan in peptide 23.

The REMD simulations of peptide 23 point to a $\beta$ VIb-like conformation similar to that of peptide 18. Again, no intramolecular hydrogen bonds were observed during the MD trajectory of peptide 23 (Figure S15). The D-Pro residue, which would be in the $i+2$ position of these $\beta$-turns, sampled the region corresponding to $\Phi=75^{\circ}, \Psi=-160^{\circ}$, which is exactly the mirror image of those described for $\beta$ Vlb-turns $\left(\Phi=-75^{\circ}\right.$, $\left.\Psi=160^{\circ}\right)^{[17]}$. D-Trp residues sampled the region corresponding to $\Phi=150^{\circ}, \Psi=-150^{\circ}$, which are close to the canonical values 
$\left(\Phi=-135^{\circ}, \Psi=135^{\circ}\right)^{[17]}$ described for residue $\mathrm{i}+1$ of $\beta \mathrm{VIb}$ turns

(Figure S16).

Interestingly, REMD showed a major population sampling a Trp side chain $x^{1} \sim 180^{\circ}$ (Figure $4 \mathrm{c}$ ) that perfectly correlates with the trans rotamer suggested by the experimental results. This rotamer would place the Trp and Pro side chains in close proximity (Figure $4 \mathrm{~d}$ ). Such proximity may explain the unusual chemical shifts of the Pro proton resonances caused by the presence of $\mathrm{CH}-\pi$ interactions, which may stabilize the whole conformation of this peptide.

A summary of the results obtained for the most representative peptides is shown in Table 3. We have demonstrated the feasibility of tuning the conformation of cyclic hexapeptide scaffolds of the type $c(\text { Xaa-D-Pro-Yaa })_{2}$. This is achieved by changing the chemical nature and stereochemistry of the amino acids in positions Xaa and Yaa.

\section{Conclusions}

Here we show how the chemical nature and/or stereochemistry of the amino acids in cyclic hexapeptide scaffolds can modulate the conformational preferences of the backbone. By changing these two properties, we were able to obtain various $\beta$-turn-like conformations that may be useful in the field of molecular recognition. Moreover, we reveal that the chemical nature and the stereochemistry of the amino acids present in this kind of cyclic peptide also affects the orientation of side chains, affording distinct types of stabilizing contacts between side chains, such as $\mathrm{CH}-\pi$ or cation- $\pi$ interactions. REMD simulations were able to overcome the multiple energy barriers that are needed to be surmounted to sample the diverse conformational states of cyclic hexapeptides in solution. This in silico approach also allowed us to analyze the Trp side chain $\chi 1$ angles of the studied peptides to predict possible interactions between side chains. Our results contribute to the further development of constrained cyclic peptides for molecular recognition processes. Moreover, they pave the way to create a skeletal conformational diversity, which is of great interest in the recognition of challenging protein surfaces.

\section{Supporting Information Summary}

Experimental methods, a detailed MS, CD and NMR characterization of peptides, and structural models and RMED analysis of selected cyclic hexapeptides are provided in the Supporting Information.

\section{Acknowledgements}

We thank the Mass Spectrometry Core Facility (IRB Barcelona), especially Marta Vilaseca and Maria del Mar Vilanova; and the NMR facility from Scientific and Technological Centre of the University of Barcelona (CCiT UB), especially Margarida Gairí, for technical support. We are also grateful to Pol Arranz-Gibert for help with the synthesis of cyclic peptides. This study was funded by the Ministry of Economy and Competitiveness (MINECO), the European Fund for Regional Development (FEDER; BIO 2016- 75327-R) and the Generalitat de Catalunya (XRB and 2014SGR-535). S.C. holded a La Caixa/IRB Barcelona Ph.D. fellowship.

Table 3. Summary of the conformational preferences of a representative set of peptides.

\begin{tabular}{|c|c|c|c|c|c|c|}
\hline \multirow[b]{2}{*}{ Peptide } & \multirow{2}{*}{$\begin{array}{c}\text { Sequence } \\
c(\text { Xaa-D-Pro-Yaa })_{2}\end{array}$} & \multirow{2}{*}{$\begin{array}{l}\text { Xaa-D-Pro } \\
\text { bond major } \\
\text { conformation }\end{array}$} & \multirow{2}{*}{$\begin{array}{c}\beta \text {-turn } \\
\text { type like }\end{array}$} & \multicolumn{3}{|c|}{ Trp side chain } \\
\hline & & & & Rotation & $\begin{array}{l}\text { Intramolecular } \\
\text { interactions }\end{array}$ & Major rotamer \\
\hline 3 & $c(\text { Arg-D-Pro-Trp })_{2}$ & trans/trans & II' & restricted & cation-тा & gauche $(+)$ / gauche (-) \\
\hline 7 & c(Glu-D-Pro-Trp $)_{2}$ & trans/trans & II' & restricted & $\mathrm{CH}-\pi$ & gauche $(+)$ \\
\hline 16 & $c(\text { Trp-D-Pro-Arg })_{2}$ & trans/trans & II' & free & ---- & --- \\
\hline 17 & c(Glu-D-Pro-D-Trp) 2 & trans/trans & II'/ I' & free & ---- & --- \\
\hline 18 & $c(D-G l u-D-P r o-T r p) 2$ & cis/cis ( 85\%) & VIb & free & ---- & --- \\
\hline 23 & $c(\mathrm{D}-\mathrm{Trp}-\mathrm{D}-\mathrm{Pro}-\mathrm{Glu})_{2}$ & cis/cis (> 95\%) & VIb & restricted & $\mathrm{CH}-\mathrm{T}$ & trans \\
\hline
\end{tabular}


Keywords: Amino acids - conformation analysis - NMR spectroscopy $\cdot$ peptides $\cdot$ structure elucidation.

[1] a) Y. Lisha, A. Rui, L. Kun, C. Bo, W. Xinhong, Curr. Pharm. Des. 2016, 22, 4086-4093; b) D. L. Selwood, Chem. Biol. Drug. Des. 2017, 89, 164-168; c) A. Zorzi, K. Deyle, C. Heinis, Curr. Opin. Chem. Biol. 2017, 38, 24-29; d) D. Weerakkody, A. Moshnikova, N. S. El-Sayed, R. C. Adochite, G. Slaybaugh, J. Golijanin, R. K. Tiwari, O. A. Andreev, K. Parang, Y. K. Reshetnyak, Sci. Rep. 2016, 6, 31322; e) Y. S. Ong, L. Gao, K. A. Kalesh, Z. Yu, Z. Wang, C. Liu, Y. Li, H. Sun, S. S. Lee, Curr. Top. Med. Chem. 2017, 17, 2302-2318; f) C. Heinis, Nat. Chem. Biol. 2014, 10, 696-698.

[2] a) T. A. Cardote, A. Ciulli, ChemMedChem 2016, 11, 787-794; b) Z. Qian, P. G. Dougherty, D. Pei, Curr. Opin. Chem. Biol. 2017, 38, 80-86; c) A. Kawamura, M. Münzel, T. Kojima, C. Yapp, B. Bhushan, Y. Goto, A. Tumber, T. Katoh, O. N. King, T. Passioura, L. J. Walport, S. B. Hatch, S. Madden, S. Müller, P. E. Brennan, R. Chowdhury, R. J. Hopkinson, H. Suga, C. J. Schofield, Nat. Commun. 2017, 8, 14773; d) P. G. Dougherty, Z. Qian, D. Pei, Biochem. J 2017, 474, 1109-1125; e) M. D. Shortridge, M. J. Walker, T. Pavelitz, Y. Chen, W. Yang, G. Varani, ACS Chem. Biol. 2017; f) R. Sable, T. Durek, V. Taneja, D. J. Craik, S. Pallerla, T. Gauthier, S. Jois, ACS Chem. Biol. 2016, 11, 2366-2374; g) A. Tavassoli, Curr. Opin. Chem. Biol. 2017, 38, 30-35.

[3] M. R. Arkin, Y. Tang, J. A. Wells, Chem. Biol. 2014, $21,1102-1114$

[4] a) A. E. Modell, S. L. Blosser, P. S. Arora, Trends Pharmacol. Sci. 2016, 37, 702-713; b) D. E. Scott, A. R. Bayly, C. Abell, J. Skidmore, Nat. Rev. Drug Discovery 2016, 15, 533-550.

[5] A. D. Cunningham, N. Qvit, D. Mochly-Rosen, Curr. Opin. Struct. Biol. 2017, 44, 59-66.

[6] a) A. Tapeinou, M.-T. Matsoukas, C. Simal, T. Tselios, Pept. Sci. 2015, 104, 453-461; b) S. H. Joo, Biomol. Ther. 2012, 20, 19-26; c) P. Thapa, M. J. Espiritu, C. Cabalteja, J. P. Bingham, Int. J. Pept. Res. Ther. 2014, 20, 545-551.

[7] C. Mas-Moruno, F. Rechenmacher, H. Kessler, Anticancer Agents Med. Chem. 2010, 10, 753-768.

[8] M. Gurrath, G. Müller, H. Kessler, M. Aumailley, R. Timpl, Eur. J. Biochem. 1992, 210, 911-921.

[9] Á. Roxin, G. Zheng, Future Med. Chem. 2012, 4, $1601-1618$.

[10] N. Bayó-Puxan, R. Rodríguez-Mias, M. Goldflam, M. Kotev, S. Ciudad, C. J. Hipolito, M. Varese, H. Suga, R. Campos-Olivas, X. Barril, V. Guallar, M. Teixidó, J. García, E. Giralt, ChemMedChem 2016, 11, 928939.

[11] X. Vila-Farrés, R. Parra-Millán, V. SánchezEncinales, M. Varese, R. Ayerbe-Algaba, N. Bayó, S. Guardiola, M. E. Pachón-Ibáñez, M. Kotev, J. García,
M. Teixidó, J. Vila, J. Pachón, E. Giralt, Y. Smani, Sci. Rep. 2017, 7, 14683.

[12] a) A. K. Kulkarni, R. P. Ojha, J. Biomol. Struct. Dyn 2015, 33, 1850-1865; b) S. M. McHugh, H. Yu, D. P. Slough, Y. S. Lin, Phys. Chem. Chem. Phys. 2017, 19, 3315-3324.

[13] a) C. Ramakrishnan, p. K. C. Paul, K. Ramnarayan, J. Biosci. 1985, 8, 239-251; b) L. M. Gierasch, C. M. Deber, V. Madison, C.-H. Niu, E. R. Blout, Biochemistry 1981, 20, 4730-4738.

[14] K. D. Kopple, T. J. Schamper, A. Go, J. Am. Chem. Soc. 1974, 96, 2597-2605.

[15] B. Guixer, X. Arroyo, I. Belda, E. Sabidó, M. Teixidó, E. Giralt, J. Pept. Sci. 2016, 22, 577-591.

[16] a) R. Fasan, R. L. Dias, K. Moehle, O. Zerbe, J. W. Vrijbloed, D. Obrecht, J. A. Robinson, Angew. Chem. Int. Ed. 2004, 43, 2109-2112; b) J. W. Bean, K. D. Kopple, C. E. Peishoff, J. Am. Chem. Soc. 1992, 114, 5328-5334.

[17] M. Schubert, D. Labudde, H. Oschkinat, P. Schmieder, J. Biomol. NMR 2002, 24, 149-154.

[18] H. K. Ganguly, B. Majumder, S. Chattopadhyay, P. Chakrabarti, G. Basu, J. Am. Chem. Soc. 2012, 134, 4661-4669.

[19] a) C. M. Venkatachalam, Biopolymers 1968, 6, 14251436; b) J. S. Richardson, Adv. Protein Chem. 1981, 34, 167-339; c) E. G. Hutchinson, J. M. Thornton, Protein Sci. 1994, 3, 2207-2216.

[20] A. Stefanucci, F. Pinnen, F. Feliciani, I. Cacciatore, G. Lucente, A. Mollica, Int. J. Mol. Sci. 2011, 12, 2853.

[21] M. W. MacArthur, J. M. Thornton, J. Mol. Biol. 1991, 218, 397-412.

[22] S. M. McHugh, J. R. Rogers, H. Yu, Y. S. Lin, J. Chem. Theory Comput. 2016, 12, 2480-2488.

[23] M. Malešević, M. Schumann, G. Jahreis, G. Fischer, C. Lücke, ChemBioChem 2012, 13, 2122-2127.

[24] a) L. J. Blair, J. D. Baker, J. J. Sabbagh, C. A. Dickey, J. Neurochem. 2015, 133, 1-13; b) M. A. Rostam, T. J. Piva, H. B. Rezaei, D. Kamato, P. J. Little, W. Zheng, N. Osman, Clin. Exp. Pharmacol. Physiol. 2015, 42, 117-124; c) W. Bedewy, H. Liao, N. A. Abou-Taleb, S. F. Hammad, T. Nasr, D. Pei, Org. Biomol. Chem. 2017, 15, 4540-4543; d) F. Mantovani, A. Zannini, A. Rustighi, G. Del Sal, Biochim. Biophys. Acta, Gen. Subj. 2015, 1850, 2048-2060.

[25] Y. J. Lin, L. K. Chu, J. C. Horng, J. Phys. Chem. B 2015, 119, 15796-15806. 


\section{FULL PAPER}

Structural tuning of cyclic hexapeptides: we have found that conformational diversity in symmetric cyclic hexapeptides can be modulated by changing the chemical nature and stereochemistry of their residues. Using NMR and MD, we have extensively analysed the conformational preferences of a family of cyclic hexapeptides.

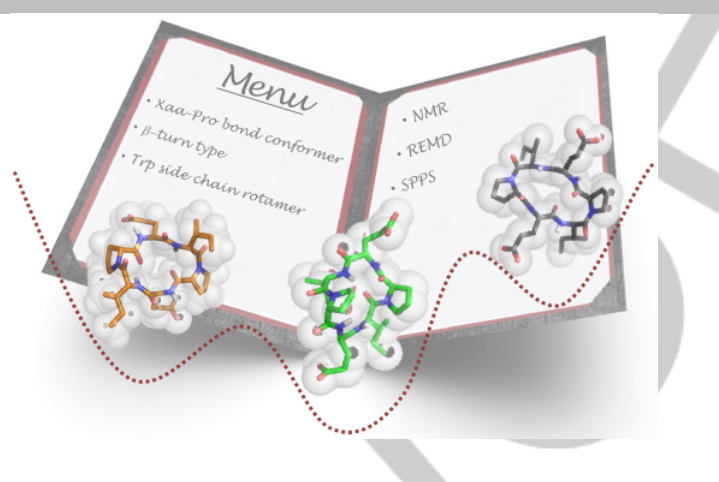

Sonia Ciudad, Núria Bayó-Puxán, Monica Varese, Jesús Seco, Meritxell Teixidó, Jesús García and Ernest Giralt*

'À la carte' cyclic hexapeptides: fine tuning conformational diversity while preserving the peptide scaffold 